\title{
Nanomedicine, an emerging therapeutic strategy for oral cancer therapy
}

Sabrina Marcazzan, DVM, PhD student ${ }^{\mathrm{a}, \mathrm{b}}$, Elena Maria Varoni, DDS, $\mathrm{PhD}^{\mathrm{b}}$, Elvin Blanco, $\mathrm{PhD}^{\mathrm{a}}$, Giovanni Lodi, DDS, $\mathrm{PhD}^{\mathrm{b}^{*}}$ and Mauro Ferrari, $\mathrm{PhD}^{\mathrm{a}, \mathrm{c}^{*}}$

* co-shared authorship

${ }^{a}$ Department of Nanomedicine, Houston Methodist Research Institute, 6670 Bertner Avenue, Houston, TX, 77030, USA. Electronic addresses: smarcazzan@houstonmethodist.org; eblanco@ houstonmethodist.org

${ }^{\mathrm{b}}$ Dipartimento di Scienze Biomediche, Chirurgiche e Odontoiatriche, Università degli Studi di Milano, Beldiletto Street 1/3, Milan, 20142, Italy. Electronic addresses: elena.varoni@unimi.it; giovanni.lodi@unimi.it

${ }^{c}$ Department of Medicine, Weill Cornell Medicine, 1300 York Avenue, New York, NY 10065, USA. Electronic address: mferrari@ houstonmethodist.org

Corresponding Author:

Prof. Giovanni Lodi

Dipartimento di Scienze Biomediche, Chirurgiche e Odontoiatriche

Università degli Studi di Milano

Beldiletto Street 1/3, Milan, 20142, Italy

Tel. + 390250319021

Fax. +390250319041

Email: giovanni.lodi@unimi.it

Co-corresponding Author:

Dr. Sabrina Marcazzan

Department of Nanomedicine

Houston Methodist Research Institute

6670 Bertner Avenue Street, R8-460, Houston, TX, 77030

Tel +1 7134417831

Email: smarcazzan@houstonmethodist.org 
Conflict of Interest Statement

None declared 
${ }^{*}$ Conflict of Interest Statement

Conflict of Interest Statement

None declared. 


\section{Introduction}

Squamous Cell Carcinoma (SCC) is the most frequently diagnosed oral cancer. It is important to note that while this term is oftentimes used to include both oral cavity and oropharyngeal SCC tumors, these are both separate entities [1, 2]. Both are included within the larger group of Head and Neck Squamous Carcinoma (HNSCC), which is the sixth most common form of cancer worldwide [3]. Herein, the term oral SCC (OSCC) is used in reference to both SCC of the oral cavity and oropharynx. OSCC represents $\sim 2-3 \%$ of all human cancers [4]. In 2012, more than 300000 and 140000 new cases of lip/oral cavity-SCC and oropharyngeal SCC were diagnosed worldwide [2]. In Western Countries, the most frequent primary sites of oral cavity SCC are the tongue and the floor of the mouth [2].

The major risk factors of OSCC include tobacco and alcohol consumption [2, 5]. Human papillomavirus (HPV) is emerging as an additional important risk factor, particularly associated with oropharyngeal SCC [2]. Oral cancerogenesis is a multistep process, in which multiple genetic and cellular alterations are involved [5]. In the oral cavity, the most common potentially malignant disorder is Oral leukoplakia [6, 7]. Imbalance of growth factors such as EGF, VEGF, PDGF, and TGF- $\beta 1$, caused by gene mutations associated with tobacco use and/or HPV 16 and 18 infection, may also contribute [2, 8-12].

Regional lymphatic metastasis and distant metastasis (DM) occurs especially in advanced stages of OSCC and correlates with a poor prognosis [8, 13, 14]. Indeed, the 5-year survival rate of OSCC in the US is $63 \%$. The survival rate has been reported to be less in the presence of lung and bone metastases [2, 14]. DM was reported in $6.6 \%$ of upper gingiva and $4.1 \%$ of tongue carcinomas [15]. However, another study reported that the majority of patients with neck lymph node metastasis had tongue cancer (69.6\%) [13]. In a larger study consisting of 502 patients affected by oral cavity SCC, only 54 patients (10.8\%) presented DMs, especially in lung, bone, and the mediastinum [14].

Treatment of OSCC is stage-dependent. Early stages treatments involve surgery or radiotherapy $(R T)$ alone. Advanced stages require surgery and/or $R T$ + chemotherapy (CT) as adjuvant treatment [2, 4, 10]. Indeed, the addition of CT significantly improved overall survival (OS) [1]. In contrast, OS was not improved in oral cavity SCC patients undergoing induction CT. However, it may result in a reduction of loco-regional recurrence [16]. The most commonly used chemotherapeutic agents are cisplatin (CDDP) and fluorouracil (5-FU), while other drugs such as carboplatin, paclitaxel, docetaxel (DTX), and methotrexate (MTX) have been reported $[1,12,17]$ Molecular-targeted agents (e.g. cetuximab and bevacizumab), gene therapies, photodynamic therapy (PDT), and immunotherapy have also been explored $[3,18,19]$. Indeed, CT results in several adverse side effects and in multi-drug resistance (MDR), which leads to a poor therapeutic response in several cases [4, 19-22]. Adverse effects of CT stem from non-specific distribution of chemotherapeutics and vary from patient to patient and type of treatment (e.g. agent, duration, dosage) [1, 20, 23]. For instance, important adverse effects of CDDP include nephrotoxicity, neurotoxicity, and gastrointestinal toxicity [23]. Also MDR contributes to CT toxicity, due to exposure of healthy cells to high levels of expelled drug $[19,20]$. The MDR mechanisms in oral cancer are still under

$$
\begin{aligned}
& \mathrm{NP}=\text { nanoparticle } \\
& \mathrm{EPR}=\text { enhanced permeability and retention } \\
& \mathrm{PLGA}=\text { poly (lactic-co-glycolic acid }) \\
& \mathrm{PEG}=\text { polyethylene glycol } \\
& \mathrm{PEI}=\text { polyethylenimine } \\
& \mathrm{CS}=\text { chitosan }
\end{aligned}
$$


investigation and contribute to failure of treatment in advanced stages of OSCC [19]. The principal mechanism consists in over-expression of multi-drug transporters, which are responsible for drug efflux from tumor cells, decreasing their efficacy $[19,20]$. Indeed, studies have reported an enhanced expression of P-gp, MDR1 and ABCG2 in cell lines resistant to CDDP, 5-FU, and in recurrent OSCC [19, 22].

To overcome the limitations of CT, nanotechnology-based systems are emerging in cancer therapy $[17,20]$. In the past decades, the intersection of nanotechnology with medicine, physics, mathematics and biology has given rise to the new field of nanomedicine. Nanoparticle (NP) systems have demonstrated high drug carrier capabilities and site-specific drug accumulation in tumors $[20,24,25]$. Several preclinical studies involving NP-based CT delivery reported higher drug solubility, drug circulation times, and efficacy when compared to conventional free drug formulations [20, 24, 26]. NPs have been reported as suitable carriers of chemopreventive compounds, nucleic acids, and diagnostic agents for both diagnostic and therapeutic (i.e. theranostic) purposes [7, 27-30]. In light of the many advantages afforded by NPs, several NP systems, such as Doxil/Caelix ${ }^{\circledR}$ and Abraxane ${ }^{\circledR}$, have been clinically approved for the treatment of certain types of cancer, such as ovarian cancer and metastatic breast cancer. Several other systems, such as gold nanoshells, are currently undergoing clinical trials [31, 32]. However, to date, no NP-based treatment has been approved for HNSCC [31, 32]. Clinical trials have been performed and other trials are currently underway to treat advanced HNSCC [31, 33, 34]. As an example, a liposomal Dox (Doxil/Caelix®) was explored in certain instances of HNSCC $[33,35,36]$.

An additional advantage of nanomedicine platforms is their versatility, and the ability to rationally design NPs to overcome sequential biological barriers (e.g. sequestration by the mononuclear phagocytic system, MDR), which have been shown to limit the efficacy of NPbased drug delivery [20, 24, 26, 31]. Indeed, the first NPs developed were able to accumulate in tumors passively through the enhanced permeability and retention effect (EPR, Fig 1) [37]. This is owed to the fact that tumor vasculature is characterized by the presence of larger endothelial fenestrations than normal endothelium, allowing for passive extravasation of NPs. However, the difference of EPR between different types of cancer may affect NP delivery to the tumor and consequently their efficacy [20,37]. Enhanced tumor targeting was obtained in second-generation NPs with the addition of components for active targeting (e.g. monoclonal antibodies) and stimulus-responsive release (e.g. pH-or hypoxia sensitive) (Fig.1) [20, 24, 25 , $31,32,38,39]$. However, none of the NPs are capable of evading the multiple biological barriers to NP drug delivery, and none of these are FDA and/or EMA approved [20, 24, 26, $31,32]$. Third-generation NPs (MSVs) consist of multistage nanovectors (MSVs). MSVs consist of first stage mesoporous silicon particles (S1MPs) and second stage-NPs embedded within nanopores (Fig. 1). Following IV administration, S1MPs are able to interact with the endothelium, allowing for release of second-stage NPs directly into the tumor interstitium [24, $39,40]$.

The aim of the present review is to summarize the current preclinical research in the field of NP-based drug delivery for OSCC therapy. Future perspectives and therapeutic strategies are also suggested.

\section{OSCC nano-based chemoprevention}

Chemoprevention for OSCC includes drugs used to reverse, prevent or suppress malignant transformation of pre-neoplastic cells [7]. In this field, natural plant compounds such as flavonoids and stilbenes have received great attention, with the majority (e.g. curcumin, naringenin) reported to show promising anti-tumor activity in preclinical studies [5, 22, 41, 42]. However, bioactive plant compounds have poor bioavailability and poor solubility, which 
reduce their efficacy in clinical studies [5, 7, 41, 43]. To overcome this problem, NP-based chemoprevention has recently been explored preclinically $[5,7,22,41,43]$.

\section{Curcumin}

NPs loaded with curcumin (Cur), a phenolic compound, were used for OSCC therapy in several studies [22, 43, 44]. PLGA NPs were shown to exert a dose- and time-dependent reduction in viability of CAL27-CDDP-resistant tongue tumor cells (CAR cells) [22]. In contrast, no significant effect was observed in normal human oral keratinocytes and gingival fibroblasts. CAR cells treated with Cur-NPs resulted in an increase of apoptosis, due to an increase of ROS production and a downregulation of multiple drug resistance protein 1 (MDR1) compared to untreated controls. Silica NPs loaded with Cur (Cur-SiNPs) had a higher cell uptake and toxicity in oral cavity SCC cells than a free Cur formulation [43]. Cur-SiNPs followed by exposure to light were also compared with a free Cur formulation followed by the same treatment [43]. Phototoxicity was significantly increased in cells treated with Cur-SiNPs compared to a free Cur formulation. Cur-SiNPs induced cell necrosis due to membrane damage, and showed a higher downregulation of NF-KB, VEGF, MMP-9, and TNF- $\alpha$ expression than a free Cur formulation. Lee et al. prepared Cur microemulsions and evaluated their cytotoxicity with and without low-frequency ultrasound on tongue cancer cells in comparison with a free Cur formulation [44]. Cur microemulsions were more cytotoxic than a free Cur formulation, and cell viability further decreased with the addition of ultrasound [44]. Mucoadhesive polycaprolactone (PCL) NPs loaded with Cur were also obtained by coating them with chitosan (CS) for local therapy of oral cavity SCC [34, 45].These Cur-NPs induced apoptotic death of tongue tumor cells and were less cytotoxic than a free Cur formulation [45].

\section{Other plant compounds}

Naringenin is a flavonoid derived from grape fruits and cherries, with proven anti-cancer effects [41]. Sulfikkarali et al. [41] prepared Naringenin-loaded NPs (NARNPs) using Eudragit $\AA$ E cationic copolymer to enhance drug solubility. The chemopreventive effect was evaluated in a DMBA-induced oral cavity SCC hamster model, which showed histopathological changes consistent with those seen in humans [46]. The oral cavity SCC group treated orally with NARNPs presented only mild dysplasia, compared to a free NAR formulation and controls. Moreover, NARNPs inhibited tumor proliferation and increased antioxidant levels compared to controls.

Gavin et al. [47] developed mucoadhesive CS-layered nanoemulsions loaded with genistein (Gen) and processed them into buccal tablet formulations. Cytotoxicity assays on OSCC cell lines revealed significant efficacy of CS-layered Gen-nanoemulsions at early time points compared to aqueous Gen-nanoemulsions [47]. In a more recent study, Li et al. [5] evaluated the effect of salvianolic acid B (SalB) phospholipid complex-loaded NPs (SalB-PLC-NPs) on oral pre-cancerous and OSCC cell lines. SalB is a bioactive component used as an active marker for Salvia miltiorrhiza Bge (Danshen) products by the National Pharmacopoeia Council of China. Indeed, the dried root of Salvia miltiorrhiza Bge (Danshen) has been used in traditional Chinese medicine against several diseases, including cancer [48]. Different concentrations of SalB-PLC-NPs significantly inhibited cell growth in both pre-cancerous and cancer cells compared to a free drug formulation of SalB [5].

\section{Nano-based chemotherapy}

CT can prove effective in advanced stage OSCC, but there is not evidence to support its benefits as an induction treatment $[1,4,10,16]$. Moreover, drug-related adverse side effects and acquired MDR limit its efficacy. Therefore, several in vitro and in vivo studies reported the 
development of NPs loaded with chemotherapeutics such as CDDP, 5FU, and moleculartargeted agents.

\section{$5-F U$}

Zhao et al. [49] synthesized 5FU-monomeric self-assembled nucleoside NPs (5-FU-SNNP) and evaluated their anti-tumor effect in vitro and in vivo. In the in vitro study, normal human oral cells were also used as controls. SNNPs exhibited low cytotoxicity for both oral cavity SCC and controls. Low toxicity of SNNPs was also reported in vivo. In a subcutaneous xenograft model of oral cavity SCC, 5-FU-SNNPs were locally injected for a duration of 3 weeks. A long-term effect of 5-FU was observed in the NP formulation compared to a free drug formulation of 5-FU. Moreover, a significant reduction in tumor growth and toxicity were reported using 5-FU-SNNPs compared to a free drug formulation of 5-FU. Similar results were obtained with a systemic treatment of 5-FU-SNNPs.

\section{CDDP}

Ligand-decorated cancer-targeted CDDP-loaded PLGA-PEG/NR7 NPs were formulated by Wang et al. [23]. NR7 peptide is based on alignment of the tripeptide motif with the EGF binding domain. Targeted NPs presented a higher uptake by oral cavity SCC cells, cytotoxicity, and cell apoptosis than non-targeted NPs and CDDP. In contrast, polymeric micelles carrying CDDP (NC-6004) exhibited lower cytotoxicity of human oral cavity SCC cell lines compared to CDDP [50]. Lower renal injury was observed in subcutaneous xenograft models treated with NC-6004 than in those treated with CDDP. Interestingly, a sublingual injection of NC-6004 significantly decreased lymphatic metastasis rates compared to CDDP in an ortothopic xenograft model of metastatic oral cavity SCC [50].

\section{Doxorubicin}

Doxorubicin (Dox) is a potent chemotherapeutic, whose cardiotoxicity limits clinical applications. Consequently, a liposomal form of Dox (Doxil( $)$ ) was developed to counter the adverse effects of the drug, indeed resulting in lessened cardiotoxicity [33, 35]. Liposomal Dox was used in clinical studies as an adjuvant chemotherapy in HNSCC [33, 35, 36, 51]. These studies reported improved results in the treatment of primary tumors, but not metastases [33, 35, 36]. Other Dox-loaded NPs have been developed for OSCC therapy. Mohan et al. [52] prepared PEGylated and non-PEGylated liposomes loaded with Dox + resveratrol (Res), a plant stilbenoid. The drug combination was more effective in HNSCC cells compared to both free and liposomal forms of single drugs. Apoptosis and DNA damage was significantly increased in the dual-drug-loaded liposome group compared to other controls. Abbasi et al. [53-55] examined stimuli-responsive cationic mesoporous silica NPs loaded with MTX and Dox on a 4-NQO-induced oral cavity SCC rat model. Both free and nanoforms of Dox combined with MTX were orally or IV administrated for 14 weeks. IV administration of MTX-Dox NPs was more effective in reducing expression of MMPs and VEGF than the oral administration, while no difference was reported regarding HER2 expression. This correlated with a lower stage of oral cavity SCC in groups treated with NPs compared to those treated with Dox and controls [53-55].

Saiyin et al. [56] loaded the autophagy inhibitor LY294002 in pH-responsive hyperbranched polyacylhydrazone (HPAH)-Dox nanomicelles. Initially, HPAH-Dox presented significantly higher cellular uptake than Dox. In subsequent experiments, addition of LY294002 to HPAHDox (LY294002-HPAH-Dox) inhibited autophagy in CAL-27 cells. This resulted in a significant increase in chemosensitivity to Dox compared to HPAH-Dox + free drug formulation of LY294002 and HPAH-Dox. 


\section{Magnetic NPs}

Due to their nano-size and the potential to modify their surface with antibodies, drugs, and MRI contrast agents, magnetic NPs have been used as theranostic agents [27-29, 34]. In addition, they are capable of converting non-ionizing electromagnetic radiation produced by an energy source (e.g. a laser) into heat energy, locally inducing apoptosis (i.e. the plasmon resonance phenomenon) [57]. Consequently, certain magnetic NPs (e.g. gold nanoshells) have been explored in clinical trials for photothermal ablation of several types of tumors, including HNSCC [31, 32, 34].

\section{Gold NPs}

Several types of gold NPs are emerging as promising drug carriers and photothermal agents [57]. Colloidal AuNPs are obtained from the reduction of $\mathrm{Au}$, and respond to exposure of near-infrared (NIR) light by converting it into heat [34, 57-59]. The efficacy of AuNPs alone or in combination with laser was investigated in a DMBA-induced oral cavity SCC hamster model [58]. In this study, AuNPs were directly injected into the tumor or in buccal pouches (controls). A significantly higher decrease in tumor growth and PCNA expression was observed in the group treated with AuNPs + laser than those treated with laser and with AuNPs only.

Iron-core NPs with a gold shell (Fe@Au) were designed more than a decade ago, to delay iron oxidation and improve its magnetic properties [29,60]. OSCC toxicity of Fe@Au was due to mitochondria-mediated autophagy, while normal oral cells were not affected [60]. Wu et al. [29] demonstrated that Fe@Au pre-cancer- and cancer-specific toxicity in vitro and in vivo correlated with release of $\mathrm{Fe}^{3+}$ ions and diminished when exposed to air for 7 days.

Gold nanoshells consisting of silica-core NPs coated with gold [34, 57, 59] were used in an unpublished clinical trial (NCT00848042) in combination with NIR photothermal therapy to treat refractory HNSCC.

Melancon et al. [38] developed gold nanoshells with a superparamagnetic iron oxide silica core (SPIO@AuNPs) and conjugated them with cetuximab (C225 monoclonal antibody). In this study, EGFR negative and EGFR overexpressing OSCC cell lines were used. Initially, binding of C225-SPIO@AuNPs to cells overexpressing EGFR was significantly higher when compared to non-targeted NPs. In a second experiment, targeted and non-targeted NPs were combined with NIR laser to kill OSCC cells, with the lowest cell viability observed in the group receiving C225-SPIO@AuNPs plus laser treatment.

\section{Other types of magnetic NPs}

In a study by Hackenberg et al. [61], UVA-1-activated zinc-oxide ( $\mathrm{ZnO})$ NPs were used alone or in combination with paclitaxel/CDDP and UVA-1 irradiation in oral cavity SCC cell lines. UVA-1 and NPs alone did not induce any significant increase in apoptosis or necrosis, compared to UVA-1 + NPs. The IC50 of both CDDP and paclitaxel did not significantly change when combined with non-irradiated NPs. In contrast, cell viability significantly decreased when chemotherapeutic agents were combined with irradiated NPs. Effective photothermal therapy in vitro (CAL-27 cells) was also obtained through combination with newly-developed cupreous complex-loaded CS (CuCC) NPs [62]. NPs in combination with laser resulted in laser power-dependent apoptosis.

In a more recent study, Lucky et al. [27] studied the biocompatibility, toxicity, and efficacy of PEGylated titanium dioxide $\left(\mathrm{TiO}_{2}\right)$ NIR excitable up-conversion NPS (UCNs) targeted against 
EGFR in a subcutaneous xenograft model of tongue cancer. UCNs were developed to increase the penetration depth of conventional PDT [27]. Targeted UCNs demonstrated higher cell uptake in vitro, resulting in higher cell death when UCNs were irradiated compared to non-targeted UCNs. In vivo toxicity studies of targeted UCNs did not reveal significant hematological or organ alterations. Efficacy studies reported that UCNs + NIR laser caused the highest tumor growth inhibition compared to NPs and PDT alone. In addition, treatment with UCNs + NIR laser resulted in larger areas of tumor necrosis and higher down-regulation of Ki67 than untreated controls [27].

\section{Magnetic NPs for other applications}

Cancer stem cells (CSCs) have high self-renewal capabilities and have been suggested to contribute to OSCC recurrence, although their characterization is still under investigation. Breast CSCs were recently targeted by AuNPs containing Dox [63]. Satapathy et al. [63] modified silver-based NPs (AgNPs) adding Quinacrine (QC), a 9-amino acridine derivative with anticancer activities, followed by encapsulation in PLGA. Interestingly, hybrid QAgNPs had different features than Ag and QC NPs (NQCs). Hybrid NPs were tested in oral cavity SCC cells, other neoplastic cell lines, and oral cavity SCC spheroids. Treatment with hybrid NPs resulted in a significant increase of pro-apoptotic protein BAX, a decrease of antiapoptotic protein BCL-XL expression, and a blocking of cell cycle in S phase compared to AgNPs and NQCs. On spheroids, hybrid NPs also induced apoptosis in CSCs by damaging DNA and inhibiting DNA repair pathways (e.g. BER). Lastly, the angiogenesis inhibition capabilities of hybrid NPs was evaluated in vivo (Chick Chorioallantoic Membrane [CAM] Assay) and compared with NQCs and AgNPs. Hybrid NPS exerted the highest antiangiogenic activity [63].

Sonodynamic therapy (SDT) consisted in replacement of light in PDT with ultrasound and is emerging as a possible treatment for oral cavity SCC [64]. Moosavi Nejad et al. [64] evaluated the effects of photocatalytic $\mathrm{TiO}_{2}$ NPs combined with high intensity focused ultrasound (HIFU) on oral cavity SCC cells and subcutaneous xenograft models. Cytotoxic effects were significantly higher in the HIFU + NPs group than HIFU or NPs alone. Upon morphological assessment, NPs significantly enhanced cell damage caused by HIFU compared to HIFU alone. In vivo, HIFU treatment with and without NPs resulted in large areas of tumor necrosis and hemorrhage. Interestingly, cell penetration of NPs in the HIFU groups was higher than that of NPs alone.

\section{Other nanotechnology-based-drug-delivery systems}

Hyper-branched polymers (HPEE) were described for the first time in PDT through the formulation of HPEE chlorine (e6) NPs [65]. The anti-proliferative effects of both HPEE-e6 NPs and free e6 were evaluated in combination with PDT on CAL-27 cells. HPEE-e6 NPs were more effective at inhibiting cell survival than free e6.

\section{Nanotechnology-based gene therapy}

Lipid-based NPs have proven to be suitable non-viral delivery vectors for gene therapy, due to their lack of immunogenicity, high tumor-specificity, and safety [66]. As a result, their use has been reported in OSCC gene therapy [21, 67].

\section{DNA transfection}

In a study by Yu et al., the anticancer effect of PEG-poly (g-benzyl-I-glutamate) (PBLG) NPs loaded with the herpes simplex virus thymidine kinase (HSV-TK) gene was evaluated in tongue cancer cells and on DMBA-induced oral cavity SCC [68]. The NPs exerted higher 
gene-transfer efficiency in vitro and DNA protection than Lipofectamine. However, their antitumor effects in vitro and in vivo were not significantly different from those of Lipofectamine [68].

Magnetic NPs have also been used as vehicles for gene therapy, to improve targeting efficiency [30]. Miao et al. [30] prepared PEl-modified $\mathrm{Fe}_{3} \mathrm{O}_{4}$ magnetic NPs to deliver a human TNF-related apoptosis-inducing ligand (TRAIL) gene driven with an hTERT tumor-specific promoter (pACTERT-TRAIL). The experiment was performed on tongue tumor cells with a magnet placed under the plate. pACTERT-TRAIL NPs demonstrated a higher transfection efficiency and induction of apoptosis than conventional PEI/lipofectin. A similar efficiency of pACTERT-TRAIL NPs was reported in a subcutaneous xenograft tongue cancer model, resulting in higher tumor growth inhibition than controls. In addition, TRAIL and TUNELpositive tumor cells were observed in treated mice compared to controls, in which tumor cells were TUNEL-negative.

\section{siRNA transfection}

In two recent studies [21, 67], lipid-calcium-phosphate NPs (LCP-NPs) were used to deliver HIF1a [67] and VEGF-A siRNA [21], respectively, in combination with photosan-mediated PDT in tongue cancer cells. Photosan is a photosensitizer drug, which is locally administered to induce neoplastic cell death through ROS production after light exposure. However, multiple treatments are often necessary for larger lesions and PDT may enhance tumor VEGF and HIF1a (hypoxia gene) expression. In both studies, LCP-NPs were actively targeted to sigma receptors $(\sigma R)$, commonly overexpressed in HNSCC and other types of tumors [21, 67]. $\sigma$ R-targeted-LCP NPs loaded with HIF1a siRNA demonstrated a higher tumor cell uptake than non-targeted NPs [67]. The combination of HIF1a siRNA LCP-NPs + PDT was more effective in reducing tumor growth than scrambled siRNA LCP + PDT in a subcutaneous xenograft tongue cancer model. Additionally, treatment with-HIF1a siRNA LCP-NPs with and without PDT resulted in a reduction of tumor size, down-regulation of angiogenic and proliferative markers, and an up-regulation of apoptosis markers [67]. Lecaros et al. [21] obtained similar results in vivo with $\sigma$ R-targeted LCP-NPs loaded with VEGF-A siRNA with and without PDT. The group with VEGF-siRNA LCP-NPs + PDT presented a greater inhibition of tumor growth and angiogenesis than scrambled siRNA LCP-NPs + PDT. As expected, VEGF-A, a-SMA, ki67, and apoptosis markers were decreased and increased in treated groups compared to PBS controls and PDT + scrambled siRNA groups.

\section{Future perspectives and strategies}

Many of the studies included herein reported the use of single entity-particles with active targeting and/or stimulus-responsive components (i.e. second-generation NPs, Fig.1) for local or systemic OSCC therapy. These systems allowed for improved solubility, drug bioavailability, increases in anti-tumor effects of PDT and chemotherapeutic agents, and reduction in toxicity. In this review, few studies used animal models and many of them used subcutaneous tumor xenograft mouse models. Despite its reproducibility, the subcutaneous tumor xenograft mouse model presents several limitations in terms of tumor location, microenvironment, vasculature, and metastatic growth, which are very different from the human disease $[46,69]$. Orthotopic models, such as direct implantation of human tumor cells into oral tissues and chemically-induced oral cavity SCC, may prove superior [69]. Since the high mortality in OSCC may be attributed to metastasis rather than the primary tumor [70], use of metastatic OSCC models may prove beneficial for development of novel therapies. Recently, Bais et al. [69], developed a metastatic model of OSCC, using human UMSCC-2 cells. Thirty-one days after cell transplantation into the tongue, metastases were observed in 
sublingual tissue, the mandible, liver, lung, and bone. Another metastatic model was also developed using the human tongue OSC-19 cell line, which resulted in lymph node metastases in 28 days [71, 72]. Herein, only one study [50] evaluated the anti-metastatic potential of NP systems on a metastatic model of OSCC.

In the clinical setting, clinical trials on NP systems combined with CT and/or RT are currently underway for treatment of recurrent and/or metastatic HNSCC [31, 32]. For instance, paclitaxel polymeric NPs in combination with CDDP is being explored for locally advanced HNSCC [31]. Among the NP systems already approved, a liposomal formulation of Dox (Doxil $\AA$ ) was used in few clinical studies in patients affected by metastatic and/or recurrent OSCC. While the response of the primary tumor to the treatment with Doxil was significant, there was no significant response of lymph node metastasis and DMs [33, 35, 36, 51].

More advanced NP platforms that may very well be applied to OSCC therapy are present only in the preclinical setting and were discussed herein. For instance, third generation NP platforms (Fig.1) showed promising results in animal models of ovarian and breast cancer [24, 40]. Single administration of MSVs loaded with nanoliposomes containing EphA2-specific siRNA resulted in a down regulation of ki67 and CD31 in an orthotopic mouse model of ovarian cancer [24]. Similarly, treatment with MSVs with two types of NPs (liposomes for siRNA delivery and PLGA-PEG NPs with DTX) reported a significant decrease of metastasis in mice bearing A375SM-Luc melanoma lung metastases compared to the combination of the two NPs without S1MPs [40].

Moreover, an injectable NP generator (iNPG) loaded with polymeric Dox (pDox) has been developed and its efficacy evaluated in an orthotopic animal model of metastatic breast cancer [26]. pDox was obtained by conjugating Dox to poly (L-glutamic acid) through a pHsensitive linkage. This platform allowed for avoidance of Dox removal via drug efflux pumps. A significant increase of median survival time $(233 \mathrm{~d})$ and a decrease in Dox-induced toxicity was obtained in a mouse model of breast cancer lung metastasis compared to free Dox (98 d) and pDox NP (99 d) treatment [26].

Nanomedicine platforms are emerging as promising therapeutic strategies for several types of cancer and their efficacy in OSCC is currently under investigation in the clinical setting. The present review highlights the current status of preclinical research in the field of nanomedicine for treatment of OSCC. The establishment and the use of more appropriate animal models of OSCC (e.g. orthotopic, metastatic models) may be suggested in order to better evaluate the therapeutic potential of NPs, particularly against metastasis. Furthermore, there is a need to assess the in vitro and in vivo efficacy of more sophisticated platforms (e.g. MSVs, iNPG) in OSCC. Indeed, new generation platforms may enhance the already proven benefits of NP based-drug delivery (e.g. overcoming chemoresistance, reducing toxicity) and concurrently address challenges such as metastasis.

\section{Acknowledgements}

M.F. is grateful for the Ernest Cockrell, Jr. Presidential Distinguished Chair at the Houston Methodist Research Institute.

\section{Funding Source}

This research did not receive any specific grant from funding agencies in the public, commercial, or not-for-profit sectors. 


\section{References:}

[1] Furness S, Glenny A-M, Worthington H V, Pavitt S, Oliver R, Clarkson JE, et al. Interventions for the treatment of oral cavity and oropharyngeal cancer: chemotherapy. In: Furness S, editor. Cochrane Database Syst. Rev., Chichester, UK: John Wiley \& Sons, Ltd; 2011, p. CD006386. doi:10.1002/14651858.CD006386.pub3.

[2] Chi AC, Day TA, Neville BW. Oral cavity and oropharyngeal squamous cell carcinoma-an update. CA Cancer J Clin 2015;65:401-21. doi:10.3322/caac.21293.

[3] Hsu H-W, Wall NR, Hsueh C-T, Kim S, Ferris RL, Chen C-S, et al. Combination antiangiogenic therapy and radiation in head and neck cancers. Oral Oncol 2014;50:19-26. doi:10.1016/j.oraloncology.2013.10.003.

[4] Naruse T, Yanamoto S, Matsushita Y, Sakamoto Y, Morishita K, Ohba S, et al. Cetuximab for the treatment of locally advanced and recurrent/metastatic oral cancer: An investigation of distant metastasis. Mol Clin Oncol 2016. doi:10.3892/mco.2016.928.

[5] Li H, Shi L, Wei J, Zhang C, Zhou Z, Wu L, et al. Cellular uptake and anticancer activity of salvianolic acid B phospholipid complex loaded nanoparticles in head and neck cancer and precancer cells. Colloids Surfaces B Biointerfaces 2016;147:65-72. doi:10.1016/j.colsurfb.2016.07.053.

[6] Li Y, Li L-J, Zhang S-T, Wang L-J, Zhang Z, Gao N, et al. In vitro and clinical studies of gene therapy with recombinant human adenovirus-p53 injection for oral leukoplakia. Clin Cancer Res 2009;15:6724-31. doi:10.1158/1078-0432.CCR-09-1296.

[7] Iriti M, Varoni E. Chemopreventive Potential of Flavonoids in Oral Squamous Cell Carcinoma in Human Studies. Nutrients 2013;5:2564-76. doi:10.3390/nu5072564.

[8] Sugiura T, Inoue $Y$, Matsuki R, Ishii K, Takahashi M, Abe M, et al. VEGF-C and VEGF$D$ expression is correlated with lymphatic vessel density and lymph node metastasis in oral squamous cell carcinoma: Implications for use as a prognostic marker. Int $\mathrm{J}$ Oncol 2009;34:673-80.

[9] Bran B, Bran G, Hörmann K, Riedel F. The platelet-derived growth factor receptor as a target for vascular endothelial growth factor-mediated anti-angiogenetic therapy in head and neck cancer. Int J Oncol 2009;34:255-61. 
[10] Keren S, Shoude Z, Lu Z, Beibei Y. Role of EGFR as a prognostic factor for survival in head and neck cancer: a meta-analysis. Tumor Biol 2014;35:2285-95. doi:10.1007/s13277-013-1303-0.

[11] Qiao B, Johnson NW, Gao J. Epithelial-mesenchymal transition in oral squamous cell carcinoma triggered by transforming growth factor- $\beta 1$ is Snail family-dependent and correlates with matrix metalloproteinase- 2 and -9 expressions. Int $\mathrm{J}$ Oncol 2010;37:663-8. doi:10.3892/ijo-00000715.

[12] de Oliveira MG, Ramalho LMP, Gaião L, Pozza DH, de Mello RA. Retinoblastoma and p53 protein expression in pre-malignant oral lesions and oral squamous cell carcinoma. Mol Med Rep 2012;6:163-6. doi:10.3892/mmr.2012.876.

[13] Hasegawa T, Shibuya Y, Takeda D, Iwata E, Saito I, Kakei Y, et al. Prognosis of oral squamous cell carcinoma patients with level IV/V metastasis: An observational study. J Craniomaxillofac Surg 2017;45:145-9. doi:10.1016/j.jcms.2016.10.011.

[14] Takahashi M, Aoki T, Nakamura N, Carreras J, Kajiwara H, Kumaki N, et al. Clinicopathological analysis of 502 patients with oral squamous cell carcinoma with special interest to distant metastasis. Tokai J Exp Clin Med 2014;39:178-85.

[15] Irani S. Distant metastasis from oral cancer: A review and molecular biologic aspects. J Int Soc Prev Community Dent 2016;6:265. doi:10.4103/2231-0762.186805.

[16] Lau A, Li K-Y, Yang W-F, Su Y-X. Induction chemotherapy for squamous cell carcinomas of the oral cavity: A cumulative meta-analysis. Oral Oncol 2016;61:104-14. doi:10.1016/j.oraloncology.2016.08.022.

[17] Calixto G, Fonseca-Santos B, Chorilli M, Bernegossi J. Nanotechnology-based drug delivery systems for treatment of oral cancer: a review. Int J Nanomedicine 2014;9:3719. doi:10.2147//JN.S61670.

[18] de Visscher SAHJ, Melchers LJ, Dijkstra PU, Karakullukcu B, Tan IB, Hopper C, et al. mTHPC-mediated Photodynamic Therapy of Early Stage Oral Squamous Cell Carcinoma: A Comparison to Surgical Treatment. Ann Surg Oncol 2013;20:3076-82. doi:10.1245/s10434-013-3006-6.

[19] Wang C, Liu XQ, Hou JS, Wang JN, Huang HZ. Molecular Mechanisms of Chemoresistance in Oral Cancer. Chin J Dent Res 2016;19:25-33. doi:10.3290/j.cjdr.a35694. 
[20] Blanco E, Shen H, Ferrari M. Principles of nanoparticle design for overcoming biological barriers to drug delivery. Nat Biotechnol 2015;33:941-51. doi:10.1038/nbt.3330.

[21] Lecaros RLG, Huang L, Lee T-C, Hsu Y-C. Nanoparticle Delivered VEGF-A siRNA Enhances Photodynamic Therapy for Head and Neck Cancer Treatment. Mol Ther 2016;24:106-16. doi:10.1038/mt.2015.169.

[22] Chang P-Y, Peng S-F, Lee C-Y, Lu C-C, Tsai S-C, Shieh T-M, et al. Curcumin-loaded nanoparticles induce apoptotic cell death through regulation of the function of MDR1 and reactive oxygen species in cisplatin-resistant CAR human oral cancer cells. Int J Oncol 2013;43:1141-50. doi:10.3892/ijo.2013.2050.

[23] Wang Z-Q, Liu K, Huo Z-J, Li X-C, Wang M, Liu P, et al. A cell-targeted chemotherapeutic nanomedicine strategy for oral squamous cell carcinoma therapy. $J$ Nanobiotechnology 2015;13:63. doi:10.1186/s12951-015-0116-2.

[24] Godin B, Tasciotti E, Liu X, Serda RE, Ferrari M. Multistage nanovectors: from concept to novel imaging contrast agents and therapeutics. Acc Chem Res 2011;44:979-89. doi:10.1021/ar200077p.

[25] lafisco M, Delgado-Lopez JM, Varoni EM, Tampieri A, Rimondini L, Gomez-Morales J, et al. Cell surface receptor targeted biomimetic apatite nanocrystals for cancer therapy. Small 2013;9:3834-44. doi:10.1002/smll.201202843.

[26] Xu R, Zhang G, Mai J, Deng X, Segura-lbarra V, Wu S, et al. An injectable nanoparticle generator enhances delivery of cancer therapeutics. Nat Biotechnol 2016;34:414-8. doi:10.1038/nbt.3506.

[27] Lucky SS, Idris NM, Huang K, Kim J, Li Z, Thong PSP, et al. In vivo Biocompatibility, Biodistribution and Therapeutic Efficiency of Titania Coated Upconversion Nanoparticles for Photodynamic Therapy of Solid Oral Cancers. Theranostics 2016;6:1844-65. doi:10.7150/thno.15088.

[28] Virupakshappa B. Applications of nanomedicine in oral cancer. Oral Health Dent Manag 2012;11:62-8.

[29] Wu Y-N, Chen D-H, Shi X-Y, Lian C-C, Wang T-Y, Yeh C-S, et al. Cancer-cell-specific cytotoxicity of non-oxidized iron elements in iron core-gold shell NPs. Nanomedicine Nanotechnology, Biol Med 2011;7:420-7. doi:10.1016/j.nano.2011.01.002. 
[30] Miao L, Liu C, Ge J, Yang W, Liu J, Sun W, et al. Antitumor Effect of TRAIL on Oral Squamous Cell Carcinoma using Magnetic Nanoparticle-Mediated Gene Expression. Cell Biochem Biophys 2014;69:663-72. doi:10.1007/s12013-014-9849-z.

[31] Anselmo AC, Mitragotri S. Nanoparticles in the clinic. Bioeng Transl Med 2016;1:10-29. doi:10.1002/btm2.10003.

[32] Wicki A, Witzigmann D, Balasubramanian V, Huwyler J. Nanomedicine in cancer therapy: Challenges, opportunities, and clinical applications. J Control Release 2015;200:138-57. doi:10.1016/j.jconrel.2014.12.030.

[33] Harrington KJ, Lewanski C, Northcote AD, Whittaker J, Peters AM, Vile RG, et al. Phase II study of pegylated liposomal doxorubicin (Caelyx) as induction chemotherapy for patients with squamous cell cancer of the head and neck. Eur J Cancer 2001;37:2015-22.

[34] Gharat SA, Momin M, Bhavsar C. Oral Squamous Cell Carcinoma: Current Treatment Strategies and Nanotechnology-Based Approaches for Prevention and Therapy. Crit Rev Ther Drug Carrier Syst 2016;33:363-400. doi:10.1615/CritRevTherDrugCarrierSyst.2016016272.

[35] Caponigro F, Comella P, Budillon A, Bryce J, Avallone A, De Rosa V, et al. Phase I study of Caelyx (doxorubicin HCL, pegylated liposomal) in recurrent or metastatic head and neck cancer. Ann Oncol Off J Eur Soc Med Oncol 2000;11:339-42.

[36] Faivre S, Alsabe H, Djafari L, Janot F, Julieron M, Domenge C, et al. Locoregional effects of pegylated liposomal doxorubicin (Caelyx) in irradiated area: a phase I-II study in patients with recurrent squamous cell carcinoma of the head and neck. Eur $\mathrm{J}$ Cancer 2004;40:1517-21. doi:10.1016/j.ejca.2004.03.018.

[37] Prabhakar U, Maeda H, Jain RK, Sevick-Muraca EM, Zamboni W, Farokhzad OC, et al. Challenges and key considerations of the enhanced permeability and retention effect for nanomedicine drug delivery in oncology. Cancer Res 2013;73:2412-7. doi:10.1158/0008-5472.CAN-12-4561.

[38] Melancon MP, Lu W, Zhong M, Zhou M, Liang G, Elliott AM, et al. Targeted multifunctional gold-based nanoshells for magnetic resonance-guided laser ablation of head and neck cancer. Biomaterials 2011;32:7600-8. doi:10.1016/j.biomaterials.2011.06.039. 
[39] Mi Y, Wolfram J, Mu C, Liu X, Blanco E, Shen H, et al. Enzyme-responsive multistage vector for drug delivery to tumor tissue. Pharmacol Res 2016;113:92-9.

doi:10.1016/j.phrs.2016.08.024.

[40] Mi Y, Mu C, Wolfram J, Deng Z, Hu TY, Liu X, et al. A Micro/Nano Composite for Combination Treatment of Melanoma Lung Metastasis. Adv Healthc Mater 2016;5:93646. doi:10.1002/adhm.201500910.

[41] Sulfikkarali N, Krishnakumar N, Manoharan S, Nirmal RM. Chemopreventive Efficacy of Naringenin-Loaded Nanoparticles in 7,12-dimethylbenz(a)anthracene Induced Experimental Oral Carcinogenesis. Pathol Oncol Res 2013;19:287-96. doi:10.1007/s12253-012-9581-1.

[42] Iriti M, Kubina R, Cochis A, Sorrentino R, Varoni EM, Kabała-Dzik A, et al. Rutin, a Quercetin Glycoside, Restores Chemosensitivity in Human Breast Cancer Cells. Phytother Res 2017. doi:10.1002/ptr.5878.

[43] Singh SP, Sharma M, Gupta PK. Enhancement of phototoxicity of curcumin in human oral cancer cells using silica nanoparticles as delivery vehicle. Lasers Med Sci 2014;29:645-52. doi:10.1007/s10103-013-1357-7.

[44] Lee M-H, Lin JL, Thomas H-W, Chen C-M, Shen W-J, Yang M-H, et al. In vitro suppression of oral squamous cell carcinoma growth by ultrasound-mediated delivery of curcumin microemulsions. Int J Nanomedicine 2012;7:941. doi:10.2147//JN.S28510.

[45] Mazzarino L, Loch-Neckel G, Bubniak LDS, Mazzucco S, Santos-Silva MC, Borsali R, et al. Curcumin-Loaded Chitosan-Coated Nanoparticles as a New Approach for the Local Treatment of Oral Cavity Cancer. J Nanosci Nanotechnol 2015;15:781-91.

[46] Mognetti B, Di Carlo F, Berta GN. Animal models in oral cancer research. Oral Oncol 2006;42:448-60. doi:10.1016/j.oraloncology.2005.07.014.

[47] Gavin A, Pham JT, Wang D, Brownlow B, Elbayoumi TA. Layered nanoemulsions as mucoadhesive buccal systems for controlled delivery of oral cancer therapeutics. Int $\mathrm{J}$ Nanomedicine 2015;10:1569-84. doi:10.2147/IJN.S75474.

[48] Zhao Y, Guo Y, Gu X. Salvianolic Acid B, a potential chemopreventive agent, for head and neck squamous cell cancer. J Oncol 2011;2011:534548. doi:10.1155/2011/534548.

[49] Zhao H, Feng H, Liu D, Liu J, Ji N, Chen F, et al. Self-Assembling Monomeric Nucleoside Molecular Nanoparticles Loaded with 5-FU Enhancing Therapeutic Efficacy against Oral Cancer. ACS Nano 2015;9:9638-51. doi:10.1021/acsnano.5b04520. 
[50] Endo K, Ueno T, Kondo S, Wakisaka N, Murono S, Ito M, et al. Tumor-targeted chemotherapy with the nanopolymer-based drug NC-6004 for oral squamous cell carcinoma. Cancer Sci 2013;104:369-74. doi:10.1111/cas.12079.

[51] Koukourakis MI, Koukouraki S, Giatromanolaki A, Archimandritis SC, Skarlatos J, Beroukas K, et al. Liposomal doxorubicin and conventionally fractionated radiotherapy in the treatment of locally advanced non-small-cell lung cancer and head and neck cancer. J Clin Oncol 1999;17:3512-21. doi:10.1200/jco.1999.17.11.3512.

[52] Mohan A, Narayanan S, Balasubramanian G, Sethuraman S, Krishnan UM. Dual drug loaded nanoliposomal chemotherapy: A promising strategy for treatment of head and neck squamous cell carcinoma. Eur J Pharm Biopharm 2016;99:73-83. doi:10.1016/j.ejpb.2015.11.017.

[53] Abbasi MM, Jahanban-Esfahlan R, Monfaredan A, Seidi K, Hamishehkar H, Khiavi MM. Oral and IV dosages of doxorubicin-methotrexate loaded- nanoparticles inhibit progression of oral cancer by down- regulation of matrix Methaloproteinase 2 expression in vivo. Asian Pac J Cancer Prev 2014;15:10705-11.

[54] Abbasi MM, Monfaredan A, Hamishehkar H, Jahanban-Esfahlan R. New formulated \&quot;DOX-MTX-loaded nanoparticles\&quot; down- regulate HER2 gene expression and improve the clinical outcome in OSCCs model in rat: the effect of IV and oral modalities. Asian Pac J Cancer Prev 2014;15:9355-60.

[55] Abbasi MM, Monfaredan A, Hamishehkar H, Seidi K, Jahanban- Esfahlan R. Novel DOX-MTX nanoparticles improve oral SCC clinical outcome by down regulation of lymph dissemination factor VEGF-C expression in vivo: oral and IV modalities. Asian Pac J Cancer Prev 2014;15:6227-32.

[56] Saiyin W, Wang D, Li L, Zhu L, Liu B, Sheng L, et al. Sequential release of autophagy inhibitor and chemotherapeutic drug with polymeric delivery system for oral squamous cell carcinoma therapy. Mol Pharm 2014;11:1662-75. doi:10.1021/mp5000423.

[57] Jain S, Hirst DG, O'Sullivan JM. Gold nanoparticles as novel agents for cancer therapy. Br J Radiol 2012;85:101-13. doi:10.1259/bjr/59448833.

[58] Afifi MM, El Sheikh SM, Abdelsalam MM, Ramadan H, Omar TA, El Tantawi M, et al. Therapeutic efficacy of plasmonic photothermal nanoparticles in hamster buccal pouch carcinoma. Oral Surg Oral Med Oral Pathol Oral Radiol 2013;115:743-51. doi:10.1016/j.0000.2012.11.020. 
[59] Hirsch LR, Gobin AM, Lowery AR, Tam F, Drezek RA, Halas NJ, et al. Metal Nanoshells. Ann Biomed Eng 2006;34:15-22. doi:10.1007/s10439-005-9001-8.

[60] Wu Y-N, Yang L-X, Shi X-Y, Li I-C, Biazik JM, Ratinac KR, et al. The selective growth inhibition of oral cancer by iron core-gold shell nanoparticles through mitochondriamediated autophagy. Biomaterials 2011;32:4565-73. doi:10.1016/j.biomaterials.2011.03.006.

[61] Hackenberg S, Scherzed A, Harnisch W, Froelich K, Ginzkey C, Koehler C, et al. Antitumor activity of photo-stimulated zinc oxide nanoparticles combined with paclitaxel or cisplatin in HNSCC cell lines. J Photochem Photobiol B Biol 2012;114:87-93. doi:10.1016/j.jphotobiol.2012.05.014.

[62] Lin M, Wang D, Liu S, Huang T, Sun B, Cui Y, et al. Cupreous Complex-Loaded Chitosan Nanoparticles for Photothermal Therapy and Chemotherapy of Oral Epithelial Carcinoma. ACS Appl Mater Interfaces 2015;7:20801-12.

doi:10.1021/acsami.5b05866.

[63] Satapathy SR, Siddharth S, Das D, Nayak A, Kundu CN. Enhancement of Cytotoxicity and Inhibition of Angiogenesis in Oral Cancer Stem Cells by a Hybrid Nanoparticle of Bioactive Quinacrine and Silver: Implication of Base Excision Repair Cascade. Mol Pharm 2015;12:4011-25. doi:10.1021/acs.molpharmaceut.5b00461.

[64] Moosavi Nejad S, Takahashi H, Hosseini H, Watanabe A, Endo H, Narihira K, et al. Acute effects of sono-activated photocatalytic titanium dioxide nanoparticles on oral squamous cell carcinoma. Ultrason Sonochem 2016;32:95-101. doi:10.1016/j.ultsonch.2016.02.026.

[65] Li P, Zhou G, Zhu X, Li G, Yan P, Shen L, et al. Photodynamic therapy with hyperbranched poly(ether-ester) chlorin(e6) nanoparticles on human tongue carcinoma CAL-27 cells. Photodiagnosis Photodyn Ther 2012;9:76-82. doi:10.1016/j.pdpdt.2011.08.001.

[66] Hattori Y, Maitani Y. Enhanced in vitro DNA transfection efficiency by novel folate-linked nanoparticles in human prostate cancer and oral cancer. J Control Release 2004;97:173-83. doi:10.1016/j.jconrel.2004.03.007.

[67] Chen W-H, Lecaros RLG, Tseng Y-C, Huang L, Hsu Y-C. Nanoparticle delivery of HIF1a siRNA combined with photodynamic therapy as a potential treatment strategy for head-and-neck cancer. Cancer Lett 2015;359:65-74. doi:10.1016/j.canlet.2014.12.052. 
[68] Yu D, Wang A, Huang H, Chen Y. PEG-PBLG nanoparticle-mediated HSV-TK/GCV gene therapy for oral squamous cell carcinoma. Nanomedicine (Lond) 2008;3:813-21. doi:10.2217/17435889.3.6.813.

[69] Bais M V., Kukuruzinska M, Trackman PC. Orthotopic non-metastatic and metastatic oral cancer mouse models. Oral Oncol 2015;51:476-82.

doi:10.1016/j.oraloncology.2015.01.012.

[70] Gohulkumar M, Gurushankar K, Rajendra Prasad N, Krishnakumar N. Enhanced cytotoxicity and apoptosis-induced anticancer effect of silibinin-loaded nanoparticles in oral carcinoma (KB) cells. Mater Sci Eng C Mater Biol Appl 2014;41:274-82. doi:10.1016/j.msec.2014.04.056.

[71] Noguchi N, Kawashiri S, Tanaka A, Kato K, Nakaya H. Effects of fibroblast growth inhibitor on proliferation and metastasis of oral squamous cell carcinoma. Oral Oncol 2003;39:240-7.

[72] Kawashiri S, Noguchi N, Tanaka A, Nakaya H, Kato K, Yamamoto E. Inhibitory effect of neoadjuvant chemotherapy on metastasis of oral squamous cell carcinoma in a mouse model. Oral Oncol 2009;45:794-7. doi:10.1016/j.oraloncology.2008.12.003.

\section{Figure Legends}

Fig.1 Different generations of nanoparticle-based drug delivery. Summary of principal features of NPs of first, second, and third generation and findings from preclinical or clinical studies in OSCC (first and second generation). 


\section{First generation}
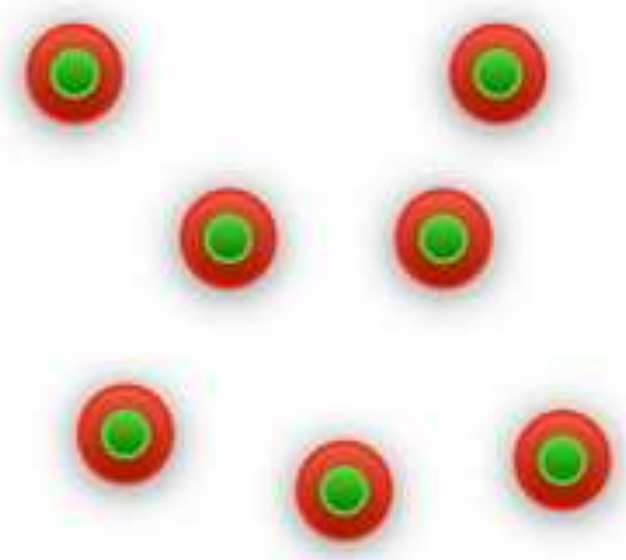

- Passive extravasation in tumors due to EPR

- Efficacy against primary tumor (Harrington et al, 2001)

- No significant effect on lymph node and distant metastasis (Harrington et al, 2001)

\section{Second generation}
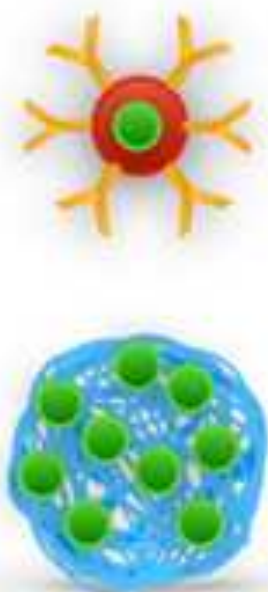

stimuli-responsive nano carriers

\section{Third generation}

multistage delivery
- Active targeting for enhanced accumulation

- Controlled release of active agents

- Efficacy against primary tumor

- Overcomes chemoresistance

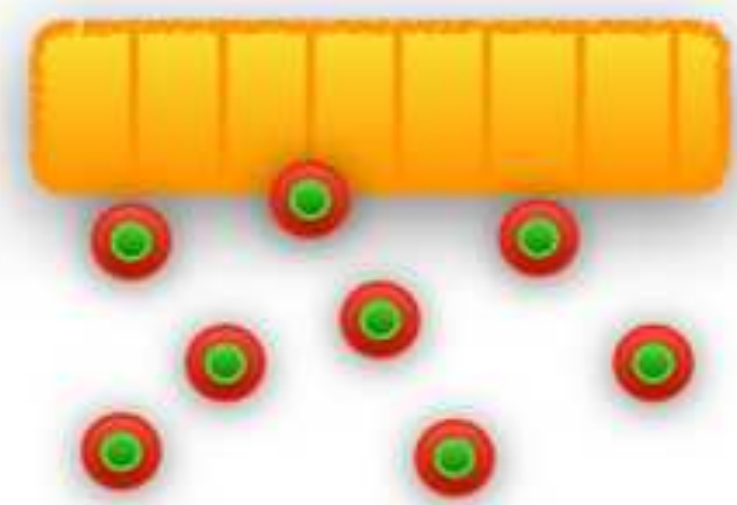

- Migration towards and adhesion to tumor endothelium and local release of NPs

- Effective against distant metastasis (e.g. lung, liver) due to organspecific delivery (Mi $\mathrm{Y}$ et al, 2016)

- Overcomes chemoresistance 